\title{
Efficacy of a Chinese Herbal Medicine, Qinzhuliangxue Granules, for The Treatment of Eczema: A Randomized, Double-Blind, Multi-Center Clinical Trial
}

\section{Ruiping Wang}

Shanghai University of Traditional Chinese Medicine Yueyang Hospital of Integrated Traditional Chinese Medicine and Western Medicine

\section{Qi Zheng}

Shanghai University of Traditional Chinese Medicine Yueyang Hospital of Integrated Traditional Chinese Medicine and Western Medicine

\section{Yu Chen}

Shanghai University of Traditional Chinese Medicine Yueyang Hospital of Integrated Traditional Chinese Medicine and Western Medicine

\section{Su Li}

Shanghai University of Traditional Chinese Medicine Yueyang Hospital of Integrated Traditional Chinese Medicine and Western Medicine

\section{Wencheng Jiang}

Shanghai Skin Diseases Hospital

\section{Qingfeng Yin}

Nanjing University of Traditional Chinese Medicine

\section{Xi Cai}

Shanghai Traditional Chinese Medicine Integrated Hospital

\section{Weian Mao}

Shanghai Seventh People's Hospital

\section{Yongmei $\mathrm{Li}$}

Longhua Hospital

\section{Huimin Zhang}

Shuguang Hospital

\section{Shaoqiong Xie}

Shanghai Skin Diseases Hospital

\section{Qinping Yang}

Huashan Hospital Fudan University

\section{Fulun Li}


Shanghai University of Traditional Chinese Medicine Yueyang Hospital of Integrated Traditional Chinese Medicine and Western Medicine

\section{Xin Li ( $\nabla 13661956326 @ 163 . c o m)$}

Shanghai University of Traditional Chinese Medicine Yueyang Hospital of Integrated Traditional Chinese Medicine and Western Medicine https://orcid.org/0000-0003-2525-9679

\section{Bin Li}

Shanghai University of Traditional Chinese Medicine Yueyang Hospital of Integrated Traditional Chinese Medicine and Western Medicine

\section{Research}

Keywords: Eczema, Qinzhuliangxue granule, Randomized double-blind trial, Eczema Area and Severity Index

Posted Date: November 2nd, 2021

DOI: https://doi.org/10.21203/rs.3.rs-989658/v1

License: (c) (i) This work is licensed under a Creative Commons Attribution 4.0 International License. Read Full License 


\section{Abstract}

Background: Eczema is the most common allergic skin disorder in the world. The treatment of eczema with western medicine generally involves antihistamines, antibiotics, glucocorticoids, and immunomodulatory preparations, which are limited by common relapse events following drug withdrawal. Many traditional Chinese medicines have demonstrated significant effects on eczema; however, high-quality clinical studies are lacking.

Objectives: We performed a multi-center, randomized, double-blind, clinical trial to evaluate the efficacy and safety of a Chinese herbal medicine, Qinzhuliangxue (QZLX) granules, and its effect on recurrence of eczema.

Methods: A total of 342 patients with eczema who met the inclusion criteria were recruited and randomly divided into a traditional Chinese medicine (TCM) treatment group, a Western medicine (WM) treatment group, and a TCM plus WM treatment group, according to random numbers generated using the central stratified zone group random method. The Eczema Area and Severity Index (EASI) score and the level of pruritus were set as the primary outcome measures, and the Dermatology Quality of Life Index (DLQI) score served as the secondary outcome measure. In this study, a two-sided p-value less than 0.05 was considered statistically significant.

Results: The median EASI score and the pruritus level at baseline were not statistically significant. However, as the treatment period progressed, the EASI score (including the total score and scores for the head, upper limb, trunk, and lower limb) and pruritus level decreased significantly in all three treatment groups. Repeated measures Analysis of Variance (ANOVA) demonstrated that the DLQI scores in the TCM, WM, and TCM+WM groups decreased significantly over time.

Limitations: Basic experiments need to be increased.

Conclusions: The Chinese herbal medicine QZLX granules significantly improved the EASI score and decreased the pruritus level in eczema patients, with good safety and no obvious adverse reactions.

\section{Trial Registration}

The protocol for this study was registered with the Clinical Trials database (NCT02517957). Registered 1 May 2015, Xia Shi Surgical Treatment for Eczema Multi-center Clinical Research - Full Text View ClinicalTrials.gov

\section{Introduction}

Eczema is the most common allergic skin disorder worldwide. The incidence of eczema in European and American countries has increased two- to three-fold in the 1997-2013 National Health Interview Survey (1). The incidence of eczema in children is as high as $10-20 \%$, while that in adults is $1-3 \%$ (2). In China, eczema is a common dermatological disease that affects a large population, resulting in serious public 
health problems and medical burden $(3,4)$. Epidemiological evidence indicates that the increased incidence of allergic diseases worldwide and the widespread use of soap and washing products has led to an increased incidence of eczema (5).

The treatment of eczema with western medicine generally involves antihistamines, antibiotics, glucocorticoids, and immunomodulatory preparations $(6,7)$. However, due to relapse events following drug withdrawal and the occurrence of adverse events, application of these drugs is limited in clinical practice (8). Thus, the development of an effective and safe treatment for eczema is important.

Numerous clinical studies on eczema treatment with Chinese herbal medicines have been performed in China (9-13). Most herbs have immunoregulatory, antimicrobial, and anti-inflammatory effects, prevent infection, restore barriers, balance Th1/Th2 cells, and regulate the production of cytokines and chemokines. Chan (14) suggested that TCM including Cortex Moutan and Herba Menthae significantly attenuate histamine release and prostaglandin D2 synthesis in rat peritoneal mast cells (RPMCs) activated by anti-IgE and compound 48/80. In contrast, Flos Lonicerae and Rhizoma Atractylodis suppress only mediator release from compound-activated RPMCs, and Cortex Phellodendri only promotes anti-IgE-induced mediator release. Our previous study summarized the clinical history of these medications, combined with domestic and foreign research areas, and discussed the mechanism of TCM treatment for patients with eczema $(15,16)$. Many TCMs have demonstrated significant effects on eczema; however, high-quality clinical studies are still limited.

QZLX granules (Table s1), a traditional herbal medicine approved by the Shanghai Food and Drug Administration in 2017 (Number: YZ170063), are composed of Hangqin, Zhenzhumu, Mudanpi, Zicao, Fangfeng, Lingcishi, Shengmuli, Shengmiren, and Shenggancao. In our previous study (Number: 07DZ19717), we demonstrated that QZLX has an anti-stress effect on xylene-induced ear swelling in mice and T cell-mediated IV type allergy, as well as a sedative effect (17). By detecting the levels of GRa receptors in the peripheral blood of eczema patients before and after treatment, and comparing with 20 healthy subjects, the team found that QZLX can upregulate the levels of GRa in the peripheral blood of eczema patients (18). This indicates that the QZLX mixture has anti-inflammatory, anti-allergic, and sedative synergistic effects on itching, and may therefore exert a therapeutic role.

Qinzhuliangxue decoction (QZLXD), an effective formula developed in the 1950 s by Xia's Chinese medical surgery (one of the most famous Shanghai styles of TCM) for eczema has been clinically used for over 50 years. Previous studies have reported that QZLXD could relieve the clinical symptoms of eczema, maintain long-term and stable efficacy, and thus improve patient quality of life (19). Although positive outcomes associated with QZLXD have been clinically observed, strong evidence is lacking. Thus, we conducted this randomized controlled trial to evaluate the efficacy and safety of QZLX granules for the clinical treatment of eczema.

\section{Materials And Methods}


A randomized, double-blind, active comparator-controlled, multi-center trial was conducted at seven medical centers in Shanghai, China. Patients were recruited and enrolled at the seven medical centers if they met the following eligibility criteria: (1) eczema patients who were diagnosed with Western medicine criteria (noncontagious inflammation of the skin, characterized chiefly by redness, itching, and an outbreak of lesions that may discharge serous matter and become encrusted and scaly) (20); (2) patients with standard syndrome of blood heat wind hyperactivity in TCM diagnosis (21); (3) aged 18-65 years old, any sex or ethnicity; (4) volunteered to participate in the clinical trial with informed consent, and could cooperate with the visit schedule; (5) lesion area of approximately $5 \%-30 \%$ of body surface area (BSA), and an Investigator Global Assessment scale of 1-3 points; (6) lesions mainly located in the trunk and limbs; and (7) women who were human chorionic gonadotrophin (HCG) negative.

\section{Ethics}

This study was approved by the Internal Review Board at the Yueyang Hospital of Integrated Chinese and Western Medicine (Approval No. 2015-015). All patients provided written informed consent prior to enrollment.

\section{Sample Size}

The target sample size for this study was based on assumptions and clinical experience from a previous study. According to the literature, the percentages of treatment effectiveness in the WM, TCM, and TCM+WM treatment groups were $63.33 \%, 83.33 \%$, and $86.67 \%$, respectively. In this study, we set the effectiveness percentage as the primary treatment effect outcome, and we assumed the following: an inspection level (a) of 0.05 and test power of 0.8 for two-sided tests, at least 94 patients required for each group, considering a $10 \%$ rate of loss to follow up, and 114 patients observed in each group, with 342 patients total between the three groups.

\section{Randomization and Blinding}

A centralized randomization system was used. Subjects were registered once they met the inclusion criteria. Basic information on the primary enrolled subjects, including date of birth, sex, ethnicity, full and abbreviated names, and predetermined prognostic factors was recorded. The subject IDs were generated after registration. Subjects were then assigned to a treatment group based on a predetermined randomized design by logging onto the randomized center website. A random coding table was established by statisticians and sealed for preservation in the Yueyang Hospital of Integrated Traditional Chinese and Western Medicine. Statisticians were blinded to the group allocation. Each subject received a medication pack containing 8 weeks of medication for all three groups, but with different medicine allocation; subjects in the TCM group took QZLX granules and a loratadine placebo orally, subjects in the WM group took placebo QZLX granules and loratadine tablets orally, and subjects in the TCM+WM treatment group took QZLX granules and loratadine tablets orally. Each pack and all medicines within it contained a label with a number on it. An emergency letter containing the patient's medication number and group was prepared for each subject for emergency unblinding. The corresponding letters were sent 
to the centers with the corresponding numbered drugs. A specially assigned person was responsible for testing the experimental indicators, while the statistician was responsible for data collection and statistical analysis.

\section{Intervention Procedure}

The intervention was divided into three stages: introduction, treatment, and follow-up.

\section{Stage 1: Introduction}

External drugs, including mometasone furoate cream (trade name: Erosone; Shanghai Schering-Plough Pharmaceutical Co., LTD., Shanghai, China), mopifloxacin ointment (trade name: Baiduan; Sino-us Tianjin Skincare Pharmaceutical Co., LTD., Tianjin, China), 3\% boric acid solution, and zinc oxide were used. Firstgeneration cephalosporin preparation was the first-choice systemic antibiotic. If a patient was allergic to cephalosporin preparations or was using cephalosporin over 7 days for disease improvement, they received clindamycin orally.

\section{Stage 2: Treatment}

The subjects were divided into three groups: TCM treatment group, WM treatment group, and TCM+WM treatment group. Subjects in the TCM group took QZLX granules and a loratadine placebo orally; subjects in the WM group took placebo QZLX granules and loratadine tablets orally, and those in the TCM+WM treatment group took QZLX granules and loratadine tablets orally.

\section{Stage 3: Follow-up}

In this stage, all the above-mentioned medications were discontinued, except for moisturizers.

\section{Observation}

The second stage of treatment lasted for 8 weeks, during which the safety indexes were observed before and after treatment. During the second stage, efficacy indexes were observed at weeks $0,2,4,6$, and 8 . The efficacy and safety of the treatment were analyzed at the end of the second stage. Complications and adverse reactions were monitored during treatment. Follow-up was performed at 2, 4, 6, and 8 weeks to assess the clinical response and rash. If eczema relapse occurred, follow-up was completed.

\section{Outcomes and Adverse Events}

The primary outcome measures were the EASI score and pruritus level (22). The secondary outcome measure was the DLQI score.

Any unexpected responses that were considered possibly or definitely related to the study treatments were reported. In the case of serious adverse events, experimental treatment was stopped immediately, 
and appropriate treatment was provided. The types and frequencies of adverse events were reported for each group.

\section{Statistical Analysis}

We applied SAS software (version 9.4; SAS Institute, Armonk, NY, USA) for data analysis. In this study, our primary analysis was a per-protocol (PP) analysis, which included 270 patients with eczema who completed the treatment and follow-up. We describe the data as means \pm standard deviations (SD) for quantitative variables with normal distribution, and as median and interquartile range (IQR) for quantitative variables with a skewed distribution. We also described the data using frequency counts and proportions (rate) for qualitative variables. Student's t-test and Mann-Whitney U tests were applied to examine the difference between quantitative variables with normal or skewed distribution, respectively, and the chi-square test was used to examine the differences between qualitative variables. Repeated measures ANOVA was applied to evaluate the efficacy of treatment, time, and the interaction between treatment and time. In this study, a two-tailed p-value $\leq 0.05$ (two-tailed) was considered statistically significant.

\section{Results}

In this study, 314 eligible patients with eczema were enrolled between August 1, 2015 and September 30, 2017. Patients were randomly assigned to receive TCM treatment $(n=102)$, WM treatment $(n=106)$, or TCM+WM treatment $(n=106)$. Among the randomized patients, $270(85.99 \%)$ completed the study (Figure 1).

Among the 270 randomized patients in the PP analysis, the median age was 53 years with an IQR of 3661 years, $55.56 \%$ of patients were male, and $87.78 \%$ were married. The mean body mass index was 22.93 , with an SD of 2.86. The median duration of eczema was 6 months, with an IQR of 0-23 months. The prevalence of concomitant diseases, including asthma, allergic rhinitis, and urticaria, was $4.07 \%$, $8.52 \%$, and $5.56 \%$, respectively. Overall, $86.30 \%$ of patients had normal electrocardiogram (ECG) results, and $96.30 \%$ were enrolled as outpatients. Baseline demographic features were similar among patients in the TCM, WM, and TCM+WM groups (Table s2), but differed between the seven clinical research centers (Table s3).

\section{Primary Outcome for Evaluation of Treatment Effect}

The EASI score was used as an index to evaluate primary outcomes. The median EASI score at baseline was 4.5 in the TCM group, 6.4 in the WM group, and 4.8 in the TCM+WM group, but the difference was not statistically significant between the three groups at baseline. Similar results were observed among the TCM, WM, and TCM+WM groups at treatment weeks 2, 4, 6, and 8, and after 2 and 4 weeks of follow-up (Table 1), although a decrease in the EASI score was used for the evaluation (Table s4). However, as treatment progressed, the EASI score (including the total score and scores for the head, upper limb, trunk, and lower limb) decreased significantly in all three groups (Table 1, Figure 2). 
Regarding the pruritus level, the median value was 2 in all three groups, both at baseline and treatment week 2 , with no statistically significant difference. Additionally, there was no statistically significant difference in the pruritus level between patients in all three groups at treatment weeks 4, 6, and 8, and after 2 and 4 weeks of follow-up (Table 1). However, as treatment progressed, the pruritus level decreased significantly in all three groups (Table 1, Figure 3).

\section{Secondary outcome for treatment effect evaluation}

The DLQI score served as the secondary outcome in this study. At baseline, the median DLQI score was 12 in the TCM group, 15 in the WM group, and 11 in the TCM+WM group, with no statistically significant difference among the three groups. The median DLQI score in all three groups was 5 at treatment week 8; however, after 2 weeks of follow-up, the median DLQI score was 1 in the TCM group, 2 in the WM group, and 1 in the TCM+WM group. Repeated measures ANOVA indicated that the DLQI score decreased significantly as treatment progressed in all three groups.(Table 1, Figure 3).

\section{Safety evaluation}

In this study, no severe treatment-related adverse events were reported, and mild treatment-related adverse events, including headache, nausea, and fatigue, occurred in $1.48 \%(4 / 270)$ of all patients, with $2.17 \%(2 / 92)$ occurring in the WM group and $2.20 \%(2 / 91)$ in the TCM+WM group. With symptomatic treatment, the four patients who experienced an adverse event recovered within 1 week.

Laboratory indices of lung and kidney function, including aspartate aminotransferase, alanine aminotransferase, urea nitrogen, serum creatinine, immunoglobulin E, red blood cell, hemoglobin, white blood cell, leukomonocyte cell, and platelet levels were analyzed to evaluate the safety of treatment. There were no statistically significant differences in any of the aforementioned lab indices among the three groups, both at baseline and after 8 weeks of treatment (Table 2, Figure s1).

\section{Discussion}

Eczema is a common, chronic, and recurrent skin disease that seriously affects the quality of life of patients, and is termed "atopic dermatitis" or "atopic eczema" in most developed countries (23). Current treatments for patients with eczema have limitations (24). For example, topical corticosteroids (25) and oral antihistamines, which are standard first-line treatments for the management of inflammatory eczema episodes, may be associated with some adverse events, including skin thinning, abnormal glucose and lipid metabolism, dry mouth, upset stomach, constipation, bone marrow suppression, and liver and kidney dysfunction $(8,26)$. With developments in medical science, research on eczema with TCM has expanded both in depth and breadth. In recent years, TCM has been discussed both in China and overseas. However, the internationalization of TCM still requires scientific verification of the research methods used in Western medicine.

At present, most hospitals treat eczema with TCM decoction, and there are fewer self-made TCM particles. However, with improvements in living standards and life rhythm speeding up, the disadvantages 
of medicinal broth are increasingly apparent, such as long decocting time, inconvenience to carry, and a lack of long-term storage. TCM granules are prepared using modern pharmaceutical technology purification and enrichment to minimize the degradation of active ingredients, and to make them lightweight and easy to carry.

Traditional Chinese medicine is an effective medical therapy system with a long history over a few thousand years. Xia's Chinese medical surgery has suggested that blood heat-induced yang floating causes eczema. Blood heat, as the name implies, refers to the heat into the blood, accelerated blood flow, vascular expansion, blood temperature rise, abnormal changes in blood function, and the pathological state of substances. Yang floats, namely erythema on the skin, papules, blisters, pruritus, and exudation represent a physical expression of blood heat. "Blood heat is the root of disease, and Yang float is the symbol of disease."(27) The theory that "expelling nearby pathogens as early as possible protects the yin fluid, and that blood heat induces yang floating"(28) has been demonstrated using Scutellaria baicalensis, mother-of-pearl, peony bark, Lithospermum, Saposhnikovia, oyster, and magnetite, with good clinical efficacy.

To evaluate the effectiveness and safety of QZLX granules for eczema treatment, we designed a randomized, double-blind, multi-center study, which was performed in seven centers in China. In this study, a granule formulation was adopted to reduce the bias of medicinal materials used in different geographical areas, varieties, and storage conditions. A placebo-controlled approach was also used to isolate the true effects and adverse reactions caused by QZLX granules, thereby minimizing subject and investigator bias. After 8 weeks of treatment, the EASI score (including the total score and scores of the head, upper limb, trunk, and lower limb) and level of pruritus decreased significantly compared to those before treatment. The DLQI score also decreased significantly over time with treatment. No serious treatment-related adverse events were reported in this study, and all laboratory indices were normal.

This study had some limitations. First, we used the PP analysis, but not the full analysis set, because 44 eczema patients did not finish the trial, which might have induced bias in the evaluation of treatment effects. Second, we did not apply a blind evaluation to estimate the effect of double-blinding in this study. Third, previous studies by our research group have confirmed that QZLX has a precise effect on eczema; however, the mechanism of action requires further study.

\section{Conclusion}

The Chinese herbal medicine QZLX granules can significantly improve the EASI score and decrease the pruritus level in eczema patients, with good safety and no obvious adverse reactions.

\section{Abbreviations}

QZLX

Qinzhuliangxue 
TCM

traditional Chinese medicine

WM

Western medicine

EASI

Eczema Area and Severity Index

DLQI

Dermatology Quality of Life Index

ANOVA

Analysis of Variance

QZLXD

Qinzhuliangxue decoction

RPMCs

rat peritoneal mast cells

BSA

body surface area

HCG

human chorionic gonadotrophin

PP analysis

per-protocol analysis

SD

standard deviations

IQR

interquartile range

ECG

electrocardiogram

\section{Declarations}

\section{Acknowledgements}

Not applicable

\section{Funding sources:}

Key project of the Shanghai Science and Technology Commission (no. 14401970200).

\section{Availability of data and materials}

I agree to share my data and materials.

Ethics approval and consent to participate 
Approval for the experimental protocol was obtained from the Internal Review Board at the Yueyang Hospital of Integrated Chinese and Western Medicine (Approval No. 2015-015). All patients provided written informed consent prior to enrollment.

\section{Consent for publication}

Not applicable

\section{Conflicts of interest}

The authors declare that there are no conflicts of interest.

\section{Author Contributions statement}

Conceptualization: L.F.L., X.L., B.L.

Data curation: R.P.W., Q.Z.

Formal analysis: R.P.W., Q.Z.

Funding acquisition: B.L., X.L.

Investigation: C.Y., L.S., W.C.J.

Methodology: B.L., X.L.

Project administration: X.L., B.L., Y.L.

Resources: X.L., B.L.

Software: Q.F.Y.

Supervision: B.L., X.L.

Validation: C.X., W.A.M., Y.M.L.

Visualization: H.M.Z., S.Q.X., Q.P.Y.

Writing - original draft preparation: Q.Z., R.P.W.

Writing - review \& editing: Q.Z., R.P.W, X.L.

\section{References}

1. Li X, Kong L, Li F, Chen C, Xu R, Wang H, et al. Association between Psoriasis and Chronic Obstructive Pulmonary Disease: A Systematic Review and Meta-analysis. PloS one. 2015;10(12):e0145221. 
2. Williams $H$, Stewart $A$, von Mutius $E$, Cookson $W$, Anderson HR. Is eczema really on the increase worldwide? The Journal of allergy and clinical immunology. 2008;121(4):947-54.e15.

3. Gu S, Yang Aw Fau - Xue CCL, Xue Cc Fau - Li CG, Li Cg Fau - Pang C, Pang C Fau - Zhang W, Zhang W Fau - Williams HC, et al. Chinese herbal medicine for atopic eczema. (1469-493X (Electronic)).

4. Hon KL, Chan Bc Fau - Leung PC, Leung PC. Chinese herbal medicine research in eczema treatment. (1749-8546 (Electronic)).

5. Kim DW, Park JY, Na GY, Lee SJ, Lee WJ. Correlation of clinical features and skin barrier function in adolescent and adult patients with atopic dermatitis. International journal of dermatology. 2006;45(6):698-701.

6. Wollenberg A, Barbarot S, Bieber T, Christen-Zaech S, Deleuran M, Fink-Wagner A, et al. Consensusbased European guidelines for treatment of atopic eczema (atopic dermatitis) in adults and children: part II. Journal of the European Academy of Dermatology and Venereology: JEADV. 2018;32(6):85078.

7. Eichenfield LF, Tom WL, Chamlin SL, Feldman SR, Hanifin JM, Simpson EL, et al. Guidelines of care for the management of atopic dermatitis: section 1. Diagnosis and assessment of atopic dermatitis. Journal of the American Academy of Dermatology. 2014;70(2):338-51.

8. Matterne U, Bohmer MM, Weisshaar E, Jupiter A, Carter B, Apfelbacher CJ. Oral H1 antihistamines as 'add-on' therapy to topical treatment for eczema. The Cochrane database of systematic reviews. 2019;1:Cd012167.

9. Chen HY, Lin YH, Hu S, Yang SH, Chen JL, Chen YC. Identifying chinese herbal medicine network for eczema: implications from a nationwide prescription database. (1741-427X (Print)).

10. Hon KL, Lo W Fau - Cheng WKF, Cheng Wk Fau - Leung T-F, Leung Tf Fau - Chow C-M, Chow Cm Fau Lau CBS, Lau Cb Fau - Fok TF, et al. Prospective self-controlled trial of the efficacy and tolerability of a herbal syrup for young children with eczema. (1471-1753 (Electronic)).

11. Huang D, Chen K, Zhang FR, Yang S, Guo Q, Xu JH, et al. Efficacy and safety of Run Zao Zhi Yang capsule on chronic eczema: a multiple-center, randomized, double-blind, placebo-controlled clinical study. (1471-1753 (Electronic)).

12. Thanik E, Wisniewski JA, Nowak-Wegrzyn A, Sampson H, Li XM. Effect of traditional Chinese medicine on skin lesions and quality of life in patients with moderate to severe eczema. (1534-4436 (Electronic)).

13. Wan HL, Chen HZ, Shi XQ. Study on effect of Traditional Chinese Medicine Jianpi Chushi decoction and ointment on chronic eczema. (2352-4146 (Electronic)).

14. Chan BC, Hon KI Fau - Leung PC, Leung Pc Fau - Sam SW, Sam Sw Fau - Fung KP, Fung Kp Fau - Lee MYH, Lee My Fau - Lau HYA, et al. Traditional Chinese medicine for atopic eczema: PentaHerbs formula suppresses inflammatory mediators release from mast cells. (0378-8741 (Print)).

15. Kuai L, Song JK, Zhang RX, Xing M, Luo Y, Ru Y, et al. Uncovering the mechanism of Jueyin granules in the treatment of psoriasis using network pharmacology. (1872-7573 (Electronic)). 
16. Liu L, Luo Y, Zhou M, Lu Y, Xing M, Ru Y, et al. Tripterygium agents for the treatment of atopic eczema: A Bayesian analysis of randomized controlled trials. (1618-095X (Electronic)).

17. Bin F, Bin L, RM J, Ning Z, YW X, Yang Y. Anti- inflammatory and Relieving Itching of Qinzhu Liangxue Mixture and Its Experimental Study. Chinese Journal of Dermatovenereology of Integrated Traditiongal and Western Medicine. 2008(02):73-5.

18. Li S CJ, Li B, et al. Effect of Qinzhu Liangxue Mixture on Eczema in Peripheral Blood of Patients with GR Receptor Alpha. Journal of Yunnan University of Traditional Chinese Medicine. 2016;39(01):1720.

19. Ma T, Chai Y, Li S, Sun X, Wang Y, Xu R, et al. Efficacy and safety of Qinzhuliangxue decoction for treating atopic eczema: a randomized controlled trial. (2224-5839 (Electronic)).

20. Rozalski M, Rudnicka L, Samochocki Z. Atopic and Non-atopic Eczema. Acta dermatovenerologica Croatica: ADC. 2016;24(2):110-5.

21. Zhang W, Leonard T, Bath-Hextall F, Chambers CA, Lee C, Humphreys R, et al. Chinese herbal medicine for atopic eczema. The Cochrane database of systematic reviews. 2005(2):Cd002291.

22. Thomas KS. EASI does it: a comparison of four eczema severity scales. The British journal of dermatology. 2015;173(2):316-7.

23. Wallach D, Taïeb A. Atopic dermatitis/atopic eczema. Chemical immunology and allergy. 2014;100:81-96.

24. Fowler E, Yosipovitch G. Chronic itch management: therapies beyond those targeting the immune system. Annals of allergy, asthma \& immunology: official publication of the American College of Allergy, Asthma, \& Immunology. 2019;123(2):158-65.

25. Lundin S, Wahlgren CF, Bergström A, Johansson EK, Dahlén E, Andersson N, et al. Use of emollients and topical glucocorticoids among adolescents with eczema: data from the population-based birth cohort BAMSE. The British journal of dermatology. 2018;179(3):709-16.

26. Rodríguez E, Baurecht $\mathrm{H}$, Herberich E, Wagenpfeil S, Brown SJ, Cordell HJ, et al. Meta-analysis of filaggrin polymorphisms in eczema and asthma: robust risk factors in atopic disease. The Journal of allergy and clinical immunology. 2009;123(6):1361-70.e7.

27. Yi R, Xun-zhe X, Le K, Dong-hui W, Qi Z, Ling-ling W, et al. Study on $\mathrm{I}^{1} \mathbf{4}^{2}$ egularity of Medication for Eczema Treated by Professor LI Bin Journal of Basic Chinese Medicine. 2018;24(12):1773-6.

28. Jing-yi Z, Bin L. Li Bin's experience in treating allergic skin diseases based on blood heat theory. Shanghai Journal of Traditional Chinese Medicine. 2013;47(10):17-8.

\section{Tables}

Table 1 Treatment responses in patients with eczema participating in a multi-center clinical research study in China in 2017 


\begin{tabular}{|c|c|c|c|c|}
\hline Effect evaluation index & $\begin{array}{l}\text { TCM } \\
(n=87)\end{array}$ & $\begin{array}{l}\text { WM } \\
(n=92)\end{array}$ & $\begin{array}{l}\text { TCM+WM } \\
(n=91)\end{array}$ & $\begin{array}{l}\mathrm{P} \text { - } \\
\text { value }\end{array}$ \\
\hline \multicolumn{5}{|c|}{ Primary outcome - EASI score, median (IQR) } \\
\hline 0 weeks & $\begin{array}{l}4.5(2.2- \\
11.6)\end{array}$ & $\begin{array}{l}6.4(3.2- \\
12.0)\end{array}$ & $\begin{array}{l}4.8(2.6- \\
11.2)\end{array}$ & 0.560 \\
\hline 2 weeks & $\begin{array}{l}3.8(1.9- \\
8.7)\end{array}$ & $\begin{array}{l}5.2(2.3- \\
9.6)\end{array}$ & $\begin{array}{l}4.0(1.9- \\
8.4)\end{array}$ & 0.310 \\
\hline 4 weeks & $\begin{array}{l}2.8(1.4- \\
6.3)\end{array}$ & $\begin{array}{l}3.9(2.0- \\
7.0)\end{array}$ & $\begin{array}{l}3.2(1.6- \\
7.2)\end{array}$ & 0.255 \\
\hline 6 weeks & $\begin{array}{l}2.3(1.1- \\
4.8)\end{array}$ & $\begin{array}{l}2.7(1.6- \\
5.7)\end{array}$ & $\begin{array}{l}2.0(1.2- \\
5.6)\end{array}$ & 0.564 \\
\hline 8 weeks & $\begin{array}{l}1.5(0.6- \\
3.6)\end{array}$ & $\begin{array}{l}2.1(0.7- \\
4.3)\end{array}$ & $\begin{array}{l}1.8(0.6- \\
4.6)\end{array}$ & 0.632 \\
\hline Follow-up 2 weeks & $\begin{array}{l}1.2(0.5- \\
3.4)\end{array}$ & $\begin{array}{l}1.4(0.5- \\
3.7)\end{array}$ & $\begin{array}{l}1.2(0.4- \\
3.8)\end{array}$ & 0.889 \\
\hline Follow-up 4 weeks & $\begin{array}{l}1.0(0.3- \\
1.8)\end{array}$ & $\begin{array}{l}0.9(0.0- \\
2.9)\end{array}$ & $\begin{array}{l}0.6(0.0- \\
3.0)\end{array}$ & 0.462 \\
\hline \multicolumn{5}{|c|}{$\begin{array}{l}\text { Primary outcome - Level of pruritus, median } \\
\text { (IQR) }\end{array}$} \\
\hline 0 weeks & $2(2-3)$ & $2(2-3)$ & $2(2-3)$ & 0.369 \\
\hline 2 weeks & $2(1-2)$ & $2(1-2)$ & $2(1-2)$ & 0.171 \\
\hline 4 weeks & $1(1-2)$ & $2(1-2)$ & $1(1-2)$ & 0.427 \\
\hline 6 weeks & $1(1-2)$ & $1(1-2)$ & $1(1-2)$ & 0.306 \\
\hline 8 weeks & $1(0-1)$ & $1(1-2)$ & $1(0-1)$ & 0.521 \\
\hline Follow-up 2 weeks & $1(0-1)$ & $1(1-1)$ & $1(0-1)$ & 0.267 \\
\hline Follow-up 4 weeks & $1(0-1)$ & $1(0-1)$ & $1(0-1)$ & 0.647 \\
\hline \multicolumn{5}{|c|}{$\begin{array}{l}\text { Secondary outcome - DLQI score, median } \\
\text { (IQR) }\end{array}$} \\
\hline 0 weeks & $12(8-20)$ & $15(8.5-22)$ & $11(9-21)$ & 0.780 \\
\hline 8 weeks & $5(2-10)$ & $5(1-10)$ & $5(1-10)$ & 0.889 \\
\hline Follow-up 2 week & $1(0-4)$ & $2(0-6)$ & $1(0-5)$ & 0.630 \\
\hline
\end{tabular}

DLQI, Dermatology Life Quality Index; EASI, Eczema Area and Severity Index; IQR, interquartile range; TCM, traditional Chinese medicine; WM, Western medicine. 
Table 2 Safety profile of traditional Chinese medicine (TCM), Western medicine (WM), and TCM+WM in patients with eczema participating in a multi-center clinical research study in China in 2017 


\begin{tabular}{|c|c|c|c|c|}
\hline $\begin{array}{l}\text { Safety outcome } \\
\text { (No. of abnormal patients) }\end{array}$ & ‡TCM $(n=87)$ & ${ }^{\dagger} W M(n=92)$ & ${ }^{\mathbb{T}} \mathrm{TCM}+\mathrm{WM}(\mathrm{n}=91)$ & §p-value \\
\hline \multicolumn{5}{|l|}{ AST, n (\%) } \\
\hline 0 weeks & $2 / 80(2.50)$ & $4 / 84(4.76)$ & $1 / 84(1.19)$ & 0.409 \\
\hline 8 weeks & $2 / 80(2.50)$ & $1 / 84(1.19)$ & $4 / 84(4.76)$ & 0.409 \\
\hline \multicolumn{5}{|l|}{ ALT, n (\%) } \\
\hline 0 weeks & $2 / 80(2.50)$ & $1 / 84(1.19)$ & $2 / 84(2.38)$ & 0.871 \\
\hline 8 weeks & $6 / 80(7.50)$ & $3 / 84(3.57)$ & $4 / 84(4.76)$ & 0.522 \\
\hline \multicolumn{5}{|l|}{ BUN, n (\%) } \\
\hline 0 weeks & $5 / 81(6.17)$ & $6 / 84(7.14)$ & $4 / 84(4.76)$ & 0.848 \\
\hline 8 weeks & $1 / 81(1.23)$ & $4 / 84(4.76)$ & $0 / 84(0.00)$ & 0.089 \\
\hline \multicolumn{5}{|l|}{ Scr, n (\%) } \\
\hline 0 weeks & $2 / 80(2.50)$ & 7/83 (8.43) & 6/84 (7.14) & 0.250 \\
\hline 8 weeks & $4 / 80(5.00)$ & $0 / 83(0.00)$ & $8 / 84(9.52)$ & 0.008 \\
\hline \multicolumn{5}{|l|}{$\lg \mathrm{E}, \mathrm{n}(\%)$} \\
\hline 0 weeks & 24/78 (30.77) & 25/81 (30.86) & 28/83 (33.73) & 0.897 \\
\hline 8 weeks & 19/78 (24.36) & 18/82 (21.95) & $20 / 84(23.81)$ & 0.945 \\
\hline \multicolumn{5}{|l|}{$\mathrm{RBC}$} \\
\hline 0 weeks & $0 / 80(0.00)$ & $2 / 83(2.41)$ & 6/84 (7.14) & 0.031 \\
\hline 8 weeks & $3 / 80(3.75)$ & $4 / 83(4.82)$ & $3 / 84(3.57)$ & 0.925 \\
\hline \multicolumn{5}{|l|}{$\mathrm{Hb}, \mathrm{n}(\%)$} \\
\hline 0 weeks & $4 / 80(5.00)$ & $6 / 83(7.23)$ & 6/84 (7.14) & 0.851 \\
\hline 8 weeks & $5 / 80(6.25)$ & $3 / 83(3.61)$ & $1 / 84(1.19)$ & 0.178 \\
\hline \multicolumn{5}{|l|}{ WBC, n (\%) } \\
\hline 0 weeks & $7 / 80(8.86)$ & 8/83 (9.64) & $5 / 84(5.95)$ & 0.674 \\
\hline 8 weeks & $5 / 80(6.25)$ & 7/83 (8.43) & 6/84 (7.14) & 0.910 \\
\hline \multicolumn{5}{|l|}{ LYMC, n (\%) } \\
\hline 0 weeks & $6 / 80(7.50)$ & 9/83 (10.84) & 7/84 (8.33) & 0.756 \\
\hline 8 weeks & $9 / 80(11.25)$ & 9/83 (10.84) & $11 / 84(13.10)$ & 0.909 \\
\hline
\end{tabular}




\begin{tabular}{|ccccc|}
\hline PLT, $\mathrm{n}(\%)$ & & & \\
\hline 0 weeks & $4 / 80(5.00)$ & $5 / 82(6.10)$ & $2 / 84(2.38)$ & 0.472 \\
\hline 8 weeks & $2 / 80(2.50)$ & $6 / 82(7.32)$ & $3 / 84(3.57)$ & 0.341 \\
\hline
\end{tabular}

‡: Data on AST, ALT, Scr, RBC, Hb, WBC, LYMC, and PLT were missing for seven patients, data on BUN were missing for six patients, and data on IgE were missing for 10 patients.

†: Data on AST, ALT, and BUN were missing for eight patients, data on Scr, RBC, Hb, WBC, and LYMC were missing for nine patients, and data on IgE and PLT were missing for 10 patients.

『: Data on AST, ALT, Scr, RBC, Hb, WBC, LYMC, PLT, BUN, and IgE were missing for seven patients.

§: P-value was based on Fisher's exact test.

ALT, alanine aminotransferase; AST, aspartate aminotransferase; BUN, urea nitrogen; Scr, serum creatinine concentration; RBC, red blood cell; WBC, white blood cell; Hb, hemoglobin; LYMC, leukomonocyte cell; PLT, platelet.

\section{Figures}




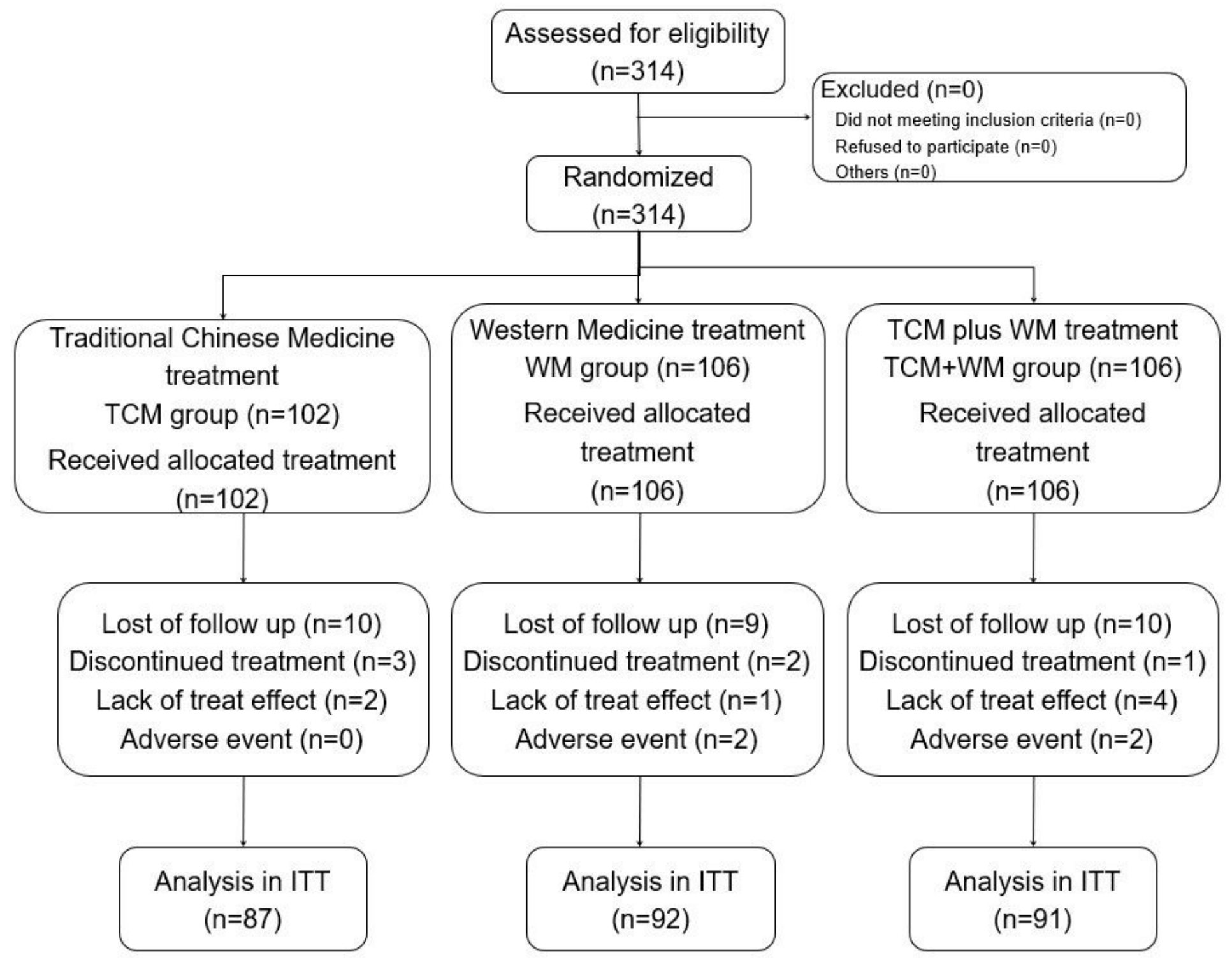

Figure 1

Consolidated Standards of Reporting Trials (CONSORT) diagram showing the flow of patients through each stage of the randomized trial. ITT, intention-to-treat. 


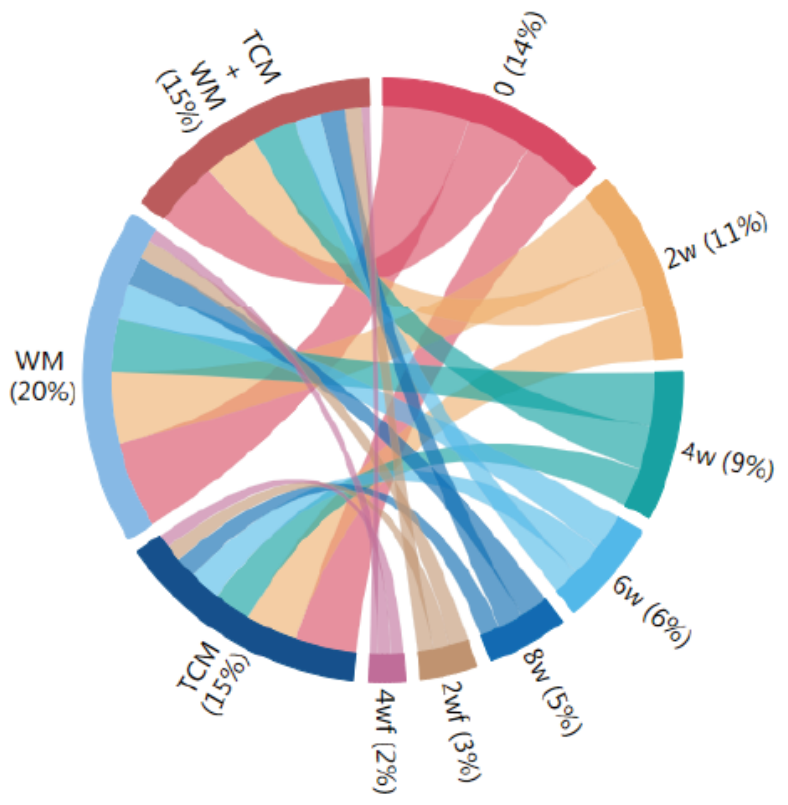

EASI total scores of eczema patients

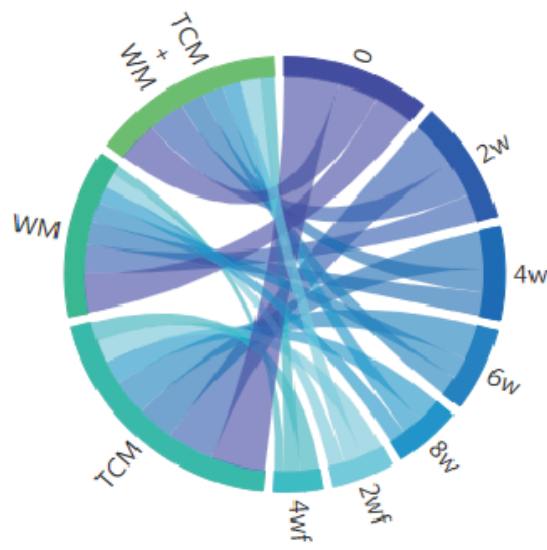

EASI scores of head

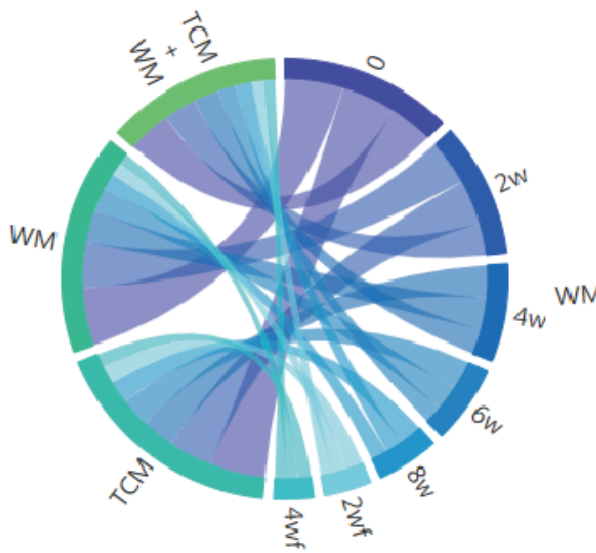

EASI scores of trunk

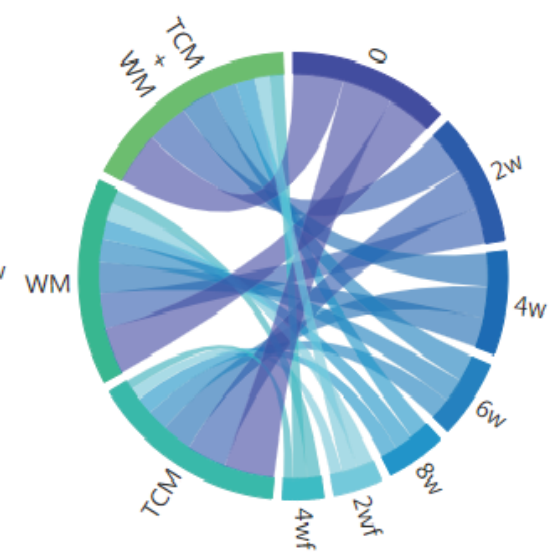

EASI scores of upper limb

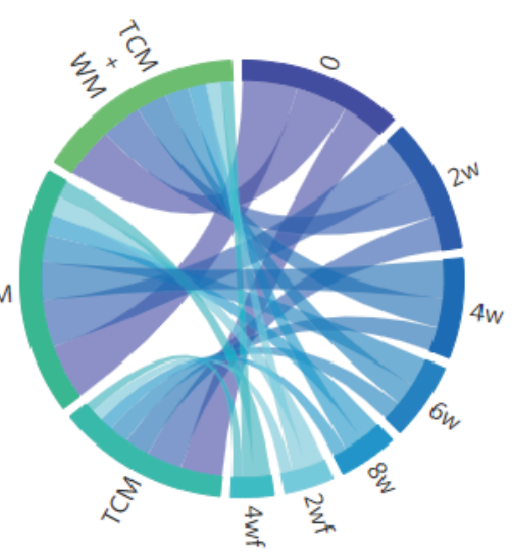

EASI scores of lower limb

\section{Figure 2}

Eczema Area and Severity Index (EASI) scores of patients with eczema at different weeks during the evaluation period, and partial evaluations of the head, upper limb, trunk, and lower limb. TCM, traditional Chinese medicine; WM, Western medicine. 


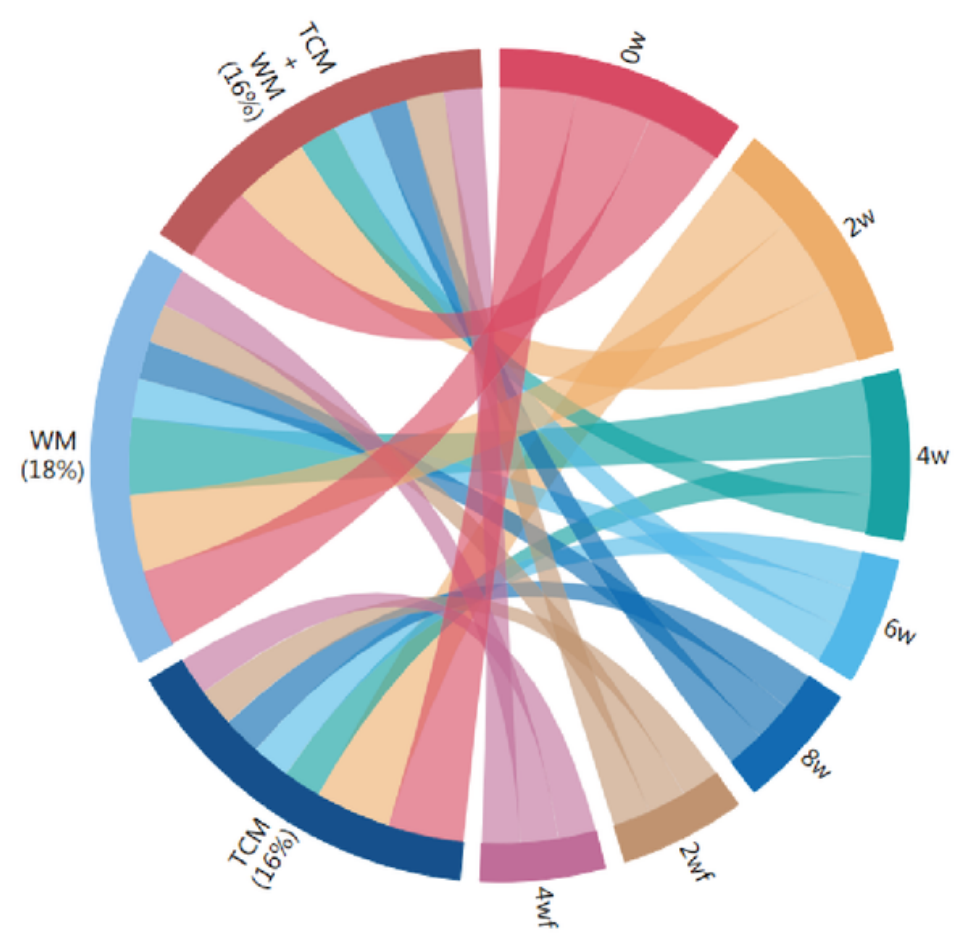

Level of pruritus among eczema patients

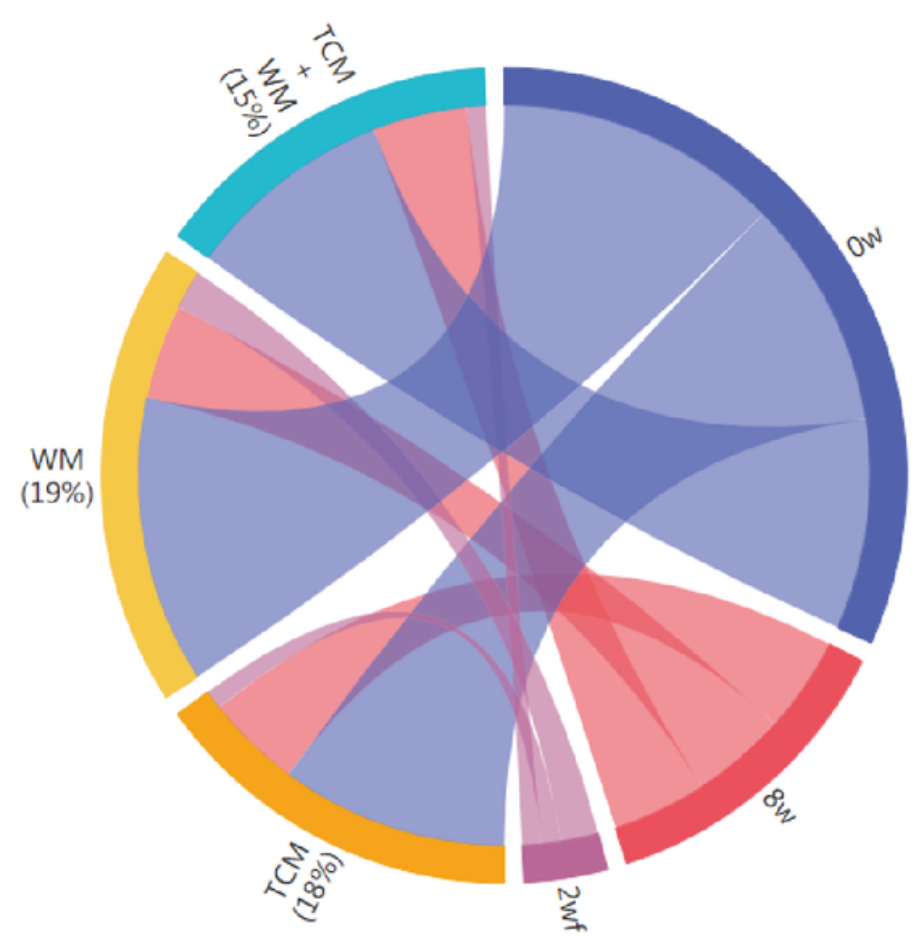

DLQI scores of eczema patients

\section{Figure 3}

Pruritus levels and Dermatology Life Quality Index (DLQI) scores at different weeks among patients with eczema. TCM, traditional Chinese medicine; WM, Western medicine.

\section{Supplementary Files}

This is a list of supplementary files associated with this preprint. Click to download.

- SuppTablesandSuppFigs.docx 